\title{
Papillary muscle dysfunction due to non-penetrating chest trauma Recognition in a potential cardiac donor
}

\author{
J. S. Schroeder, E. B. Stinson, C. P. Bieber, L. Wexler, N. E. Shumway, \\ and D. C. Harrison \\ From the Departments of Medicine, Pathology, Radiology and Surgery, \\ Stanford University Medical Center, Stanford, California, U.S.A.
}

\begin{abstract}
A previously healthy, I8-year-old man was transferred to Stanford Medical Center as a potential cardiac donor 20 hours after suffering severe head injury. Because of a systolic heart murmur, left ventricular angiography was performed and showed obvious mitral insufficiency. At necropsy the heart was normal except for haemorrhagic necrosis within the tips of the mitral papillary muscles. Thus, papillary muscle dysfunction due to intramyocardial haemorrhage must be added to the differential diagnosis of traumatic mitral insufficiency.
\end{abstract}

Non-penetrating trauma is a well-known though uncommon cause of cardiac disease. Injuries to the heart which result from blunt trauma have been extensively reviewed by Parmley, Manion, and Mattingly (1958) in their analysis of 546 necropsy cases. Documented mitral valve lesions include laceration of the leaflets and laceration or rupture of the chordae tendineae and papillary muscles. In this report we present a case of papillary muscle dysfunction secondary to blunt chest trauma, without associated injury of the myocardium.

\section{Case report}

A previously healthy, I8-year-old man was struck by an automobile and thrown into the air, suffering an extensive skull fracture. On initial evaluation he responded to painful stimulation, but rapidly progressed to a comatose state and required mechanical ventilation. Circulatory support with metaraminol was initiated 16 hours after admission because of progressive hypotension. Twenty hours after admission a diagnosis of brain death was made on the basis of the comatose state, arreflexia, apnoea, fixed and dilated pupils, absence of response to irrigation of the ear canals with hot and cold water, and isoelectric electroencephalogram, and the patient was transferred to Stanford Medical Center as a potential cardiac donor.
Cardiovascular examination revealed a blood pressure of $130 / 100 \mathrm{mmHg}$ with metaraminol infused intravenously at $50 \mu \mathrm{g} / \mathrm{min}$ and pulse of $160 / \mathrm{min}$. The central venous pressure was $0 \mathrm{~cm}$ saline while on positive pressure ventilation. There were superficial abrasions on the right side of the lower thorax. The heart sounds were within normal limits. There was a grade $2-3 / 6$ midsystolic murmur heard best at the lower left sternal border and midway to the apex. The murmur was inaudible when the systolic pressure was below 90 $\mathrm{mmHg}$.

The electrocardiogram showed sinus tachycardia with an intraventricular conduction defect and $T$ wave changes suggesting hyperkalaemia (serum potassium at the time of transfer was 8.0 $\mathrm{mEq} /$ litre). Chest $x$-rays at initial evaluation showed a normal-sized cardiac silhouette with increased pulmonary vascular markings consistent with pulmonary oedema, clearing by the time of transfer.

Left ventricular angiography was performed to determine the cause of the systolic murmur(Fig. I). Cineangiography in both oblique projections showed an intact ventricular septum. A central jet of contrast material, however, consistently refluxed through the mitral valve into a normalsized left atrium. The mitral valve leaflets were mobile, with slight prolapse of the aortic leaflet posteriorly. Biplane frontal and lateral large film angiography confirmed the mitral valve insufficiency. Both papillary muscles appeared intact. Left ventricular contractility and end-diastolic pressure were normal. 
646 Schroeder, Stinson, Bieber, Wexler, Shumway, and Harrison

FIG. I Left ventricular angiogram, lateral projection. $A P M=$ anterior papillary muscle; 2 long arrows = posterior papillary muscle; solid short arrow = anterior (aortic) leaflet of mitral valve; open short arrow $=$ posterior leaflet.

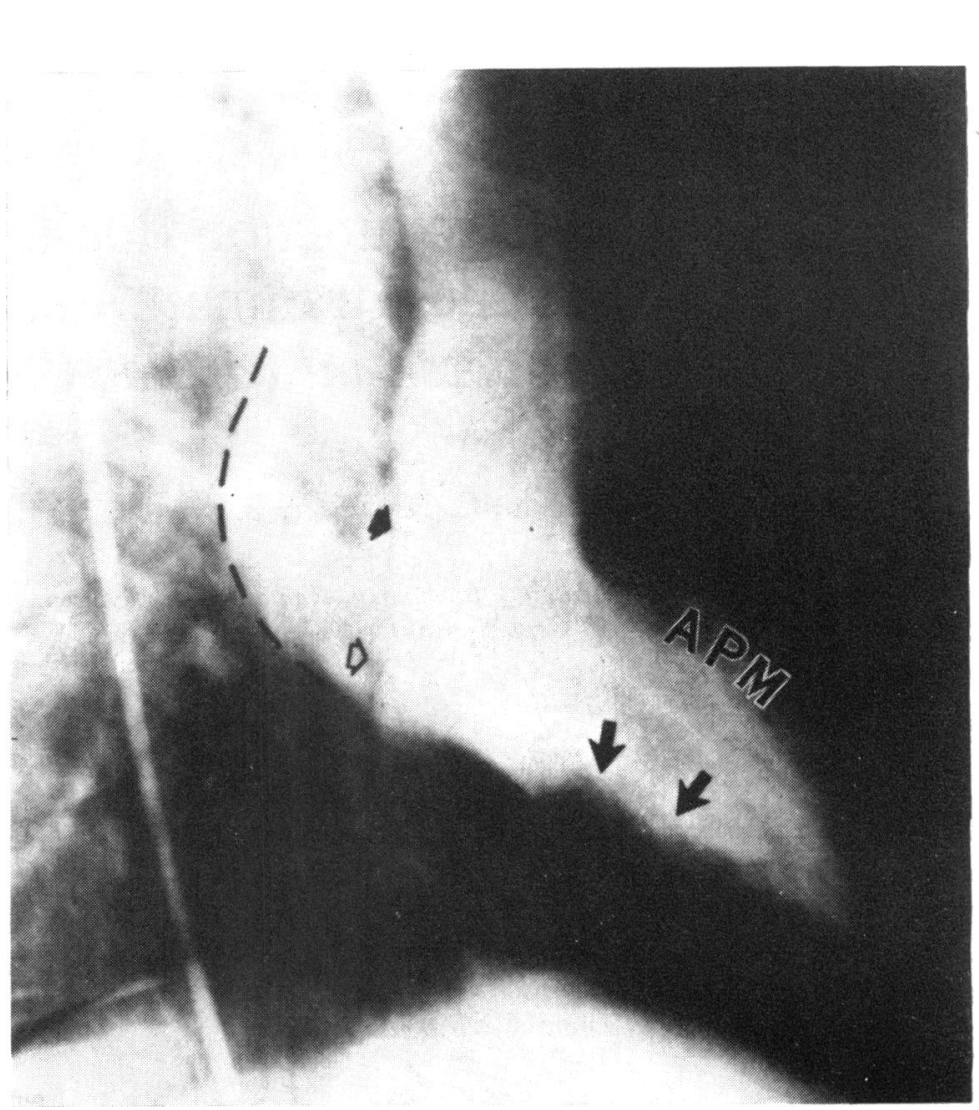

FIG. 2 Arrows point to localized areas of haemorrhage within the tips of the mitral papillary muscles.

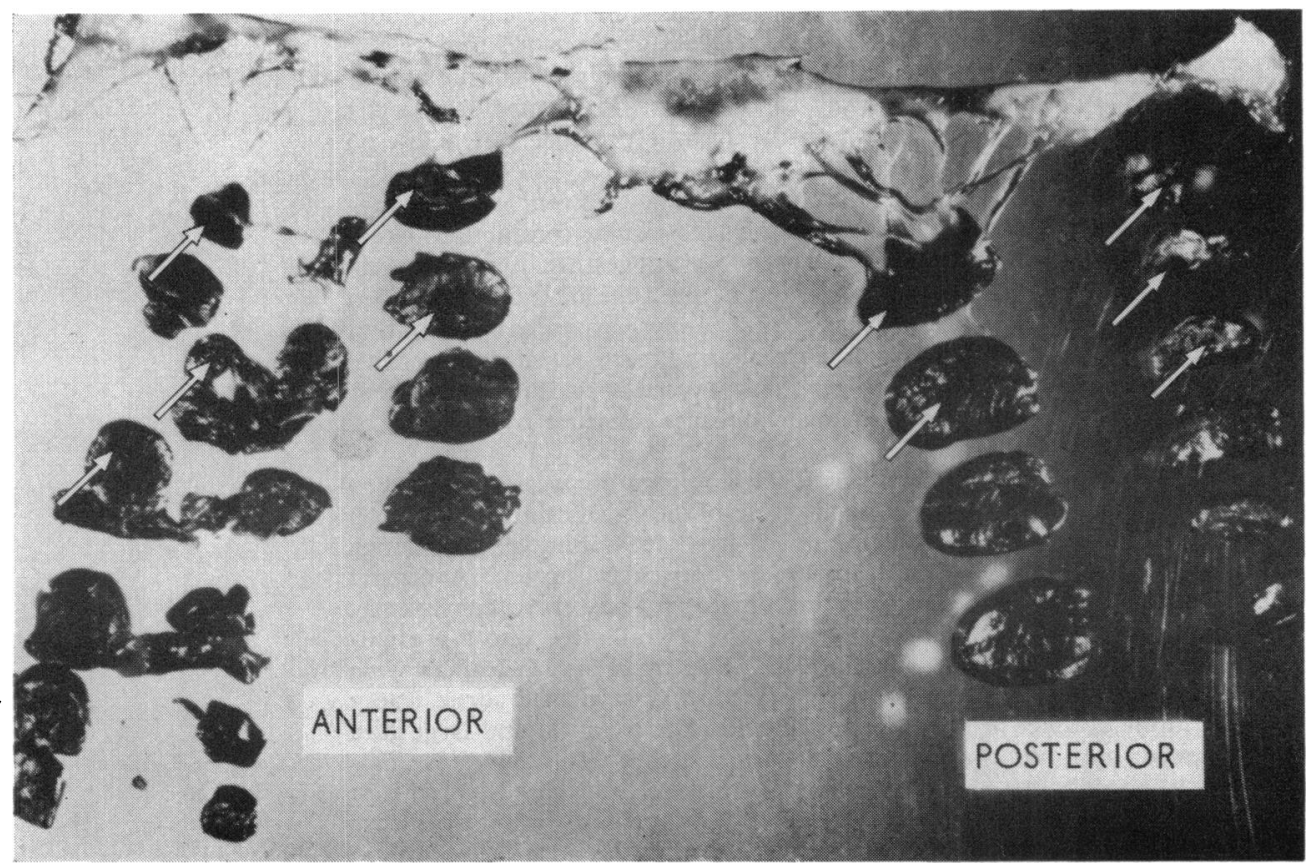


The patient was not considered a suitable organ donor and expired the following day. Necropsy confirmed massive head injury with intracerebral haemorrhage and oedema as the cause of death. The heart, weighing $305 \mathrm{~g}$, had an unremarkable gross appearance and configuration. There was no papillary muscle rupture or disruption of the chordae tendineae of the mitral valve. Sectioning of the left ventricular papillary muscles, however, disclosed localized haemorrhage within the myocardium underlying the attachments of the chordae tendineae (Fig. 2). Microscopical examination of these areas showed haemorrhage and myocardial necrosis accompanied by a polymorphonuclear infiltrate. Microscopical examination of the remainder of the heart, including the bases of the papillary muscles, the mural and septal myocardium, and the coronary arteries, was normal.

\section{Discussion}

Because of a normal heart size and variable systolic murmur, traumatic heart disease was not suspected until the patient was evaluated as a possible cardiac donor. The dynamic demonstration of severe mitral insufficiency with left ventricular angiography was critical in this assessment. Indeed, without left ventricular angiography and without evidence of significant chest trauma, it is likely that the cause of cardiac dysfunction - localized papillary muscle damage - would have gone unrecognized at necropsy.

Parmley et al. (1958) discussed the various proposed mechanisms of cardiac lesions due to blunt trauma, including blast and compressive injury. In the case described above the occurrence of a sudden concussive or compressive force at end-diastole or during isovolumic contraction may have caused sufficient stress to the mitral papillary muscles to cause intrapapillary muscle haemorrhage without rupture or tearing. The angiographic demonstration of significant mitral insufficiency is consistent with the clinical observation of obvious softening or disappearance of the systolic murmur during periods of hypotension. The localized distribution of haemorrhage within the tips of the mitral papillary muscles without evidence of general subendocardial or myocardial damage essentially rules out injury related to vasopressor administration (Szakacs and Mehlman, 1960) or intracranial injury (Koskelo, Punsar, and Sipila, 1964).

\section{References}

Koskelo, P., Punsar, S., and Sipila, W. (1964). Subendocardial haemorrhage and E.C.G. changes in intracranial bleeding. British Medical fournal, $\mathbf{r}$, I479.

Parmley, L. F., Manion, W. C., and Mattingly, T. W. (1958). Non-penetrating traumatic injury of the heart. Circulation, 18, 371 .

Szakacs, J. E., and Mehlman, B. (1960). Pathologic changes induced by I-norepinephrine. Quantitative aspects. American fournal of Cardiology, 5, 619.

Requests for reprints to Dr. Edward B. Stinson, Division of Cardiovascular Surgery, Stanford University Medical Center, Stanford, California 94305, U.S.A. 